\title{
Ship Detection in High-Resolution Dual-Polarization SAR Amplitude Images
}

\author{
Gui Gao, Gongtao Shi, and Shilin Zhou \\ School of Electronics Science and Engineering, National University of Defense Technology, Changsha, Hunan 410073, China \\ Correspondence should be addressed to Gui Gao; dellar@126.com
}

Received 31 October 2012; Accepted 8 January 2013

Academic Editor: Deren Li

Copyright (c) 2013 Gui Gao et al. This is an open access article distributed under the Creative Commons Attribution License, which permits unrestricted use, distribution, and reproduction in any medium, provided the original work is properly cited.

\begin{abstract}
A constant false alarm rate (CFAR) detecting method for ships in high-resolution dual-polarization synthetic aperture radar (SAR) amplitude images has been proposed in this paper. First, by the production of amplitude images from two polarimetric channels, a novel detector simply called the PMA detector has been constructed. We testified that the PMA detector could improve the signal-to-clutter ratio (SCR) and make the discrimination of a ship from clutter more easily. Second, the PMA detector's statistical model has been described by the well-known $\mathscr{G}^{0}$ distribution when facing complex sea background. The experiments performed on measured dual-polarization TerraSAR-X images demonstrate the good performance of the proposed CFAR detecting method.
\end{abstract}

\section{Introduction}

Ship detection in high-resolution synthetic aperture radar (SAR) images has become an increasing interest during the past several years [1]. It plays an important role for various potential applications like marine surveillance, vessel traffic control, military service, and so forth [1-3]. Additionally, with the development of sensor techniques, the advanced polarimetric SAR systems have also been validated that more completed description of target-scattering behavior could be provided than traditional single-channel SAR systems, such as $\mathrm{HH}, \mathrm{HV}$, and VV SAR, which is not sufficient for ship detection [2, 4]. Since better discriminating target signal from the surrounding clutter can be obtained by polarimetric SAR systems, ship detection in multipolarimetric or dualpolarimetric SAR images is receiving intensive attentions in present.

Nowadays, some famous contemporary satellite SAR systems, for example, TerraSAR-X, COSMO-SkyMed, and RadarSat-2, support dual-polarization acquisition mode [5]. However, the available data is, mostly, the amplitude data and not the complex-valued data [6]. It is an important data modality because of the image products provided by many satellite SAR systems, for instance, ERS. Thus, we mainly focus on ship detection using only dual-pol SAR amplitude images in this paper.

A wide variety of methods have been proposed for detecting ships in polarimetric SAR images. The basic idea is to reduce three channels of polarimetric SAR data to a single decision criterion [7]. Some popular detectors [7], including the optimal polarimetric detector (OPD), the polarimetric whitening filter (PWF), the span detector, the power maximization synthesis (PMS) detector, and, more recently, the identity likelihood ratio test (ILRT), have been developed and shown that they can perform in a way that targets are more easily discriminated from clutter. Unfortunately, other detectors mentioned earlier except for the span detector are very difficult to implement due to the missing of phase information when the only amplitude is considered [7].

The span detector is a widely used processor which is a noncoherent sum of all polarimetric channels and only makes use of image intensities or amplitudes. It has also been proven that this detector can acquire a better detection performance than $\mathrm{HH}, \mathrm{HV}$, or VV individually. Nevertheless, the expected adaptive detection threshold is impossible when facing complex clutter background by this detector, which limits the applications of this detector. 
Our goal is to present a powerful detector for ship detection in high-resolution dual-polarization SAR amplitude images. On one hand, this detector can improve the signal-to-clutter ratio (SCR) to enhance the moving targets or restrain the clutter. Meanwhile, we wish that a flexible and adaptive constant false alarm rate (CFAR) threshold could be derived from this detector. Under this consideration, this paper proposed a novel detector similarly with the span detector, simply called the product of multilook amplitudes (PMAs) detector.

\section{Dual-Polarization SAR Data Description}

The fundamental quantities measured by a polarimetric SAR are the complex scattering matrix with four elements, which, in complete form, can be expressed by [8]

$$
\mathbf{S}=\left[\begin{array}{ll}
S_{h h} & S_{h v} \\
S_{v h} & S_{v v}
\end{array}\right]
$$

where $S_{p q}$ is the scattering element with $p$ transmit and $q$ receive polarizations; $p$ and $q$ denote either horizontal $(h)$ or vertical $(v)$ polarization. In a reciprocal medium, the crosspolar elements of the scattering are identical; that is, $S_{h v}=$ $S_{v h}$. In this matter, the scattering matrix shown in (1) can be organized into a column vector

$$
\mathbf{X}=\left[\begin{array}{lll}
S_{h h} & S_{h v} & S_{v v}
\end{array}\right]^{\mathrm{T}},
$$

where the superscript " $\mathrm{T}$ ” represents transpose. The equation (2) is called single-look complex scattering vector. Based on the coherent nature of SAR, $\mathbf{X}$ follows a zero mean multivariate complex Gaussian distribution. The detailed discussion about this distribution can be found in [8] and [10].

2.1. Polarimetric Covariance Matrix for Dual-Polarization Case. For dual-polarization case, the single look scattering vector shown in (2) can be simplified to

$$
\mathbf{u}=\left[\begin{array}{ll}
S_{1} & S_{2}
\end{array}\right]^{\mathrm{T}}
$$

Herein, for convenience, we use $S_{1}$ or $S_{2}$ to indicate one of the scattering elements $S_{h h}, S_{h v}$, and $S_{v v}$ in any order, as well as $S_{1} \neq S_{2}$. Additionally, to reduce the influence of speckle, SAR data are often multi-look averaged. As the polarimetric information can also be represented by a covariance matrix, the $n$-look sample covariance matrix is defined as $[8,9]$

$$
\mathbf{R}=\frac{1}{n} \sum_{k=1}^{n} \mathbf{u}(k) \mathbf{u}(k)^{\mathrm{H}}=\frac{1}{n} \sum_{k=1}^{n}\left[\begin{array}{cc}
\left|S_{1}(k)\right|^{2} & S_{1}(k) S_{2}(k)^{*} \\
S_{1}(k)^{*} S_{2}(k) & \left|S_{2}(k)\right|^{2}
\end{array}\right],
$$

where the superscript $*$ means complex conjugate and $\mathrm{H}$ represents conjugate complex transpose, $n$ is the number of looks, and $\mathbf{u}(k)=\left[S_{1}(k), S_{2}(k)\right]^{\mathrm{T}}$ is the $k$ th singlelook image. Assuming statistical ergodicity and constant RCS background, the random matrix $\mathbf{R}$, known as the complex Wishart distribution [10], is with probability density

$$
p_{\mathbf{R}}(\mathbf{R})=\frac{n^{2 n} \operatorname{det}(\mathbf{R})^{n-2} \exp \left[-n \operatorname{Tr}\left(\mathbf{C}^{-1} \mathbf{R}\right)\right]}{\pi \Gamma(n) \Gamma(n-1) \operatorname{det}(\mathbf{C})^{n}},
$$

where $\Gamma(\cdot)$ is the gamma function, and $\operatorname{Tr}(\cdot)$ indicates the matrix trace. The symbol $\operatorname{det}(\cdot)$ denotes the determinant operator, and the covariance matrix is $2 \times 2$ complex, Hermitian, written as

$$
\begin{aligned}
\mathbf{C} & =E\left[\mathbf{u u}^{\mathrm{H}}\right] \\
& =\left[\begin{array}{cc}
E\left(\left|S_{1}\right|^{2}\right) & \sqrt{E\left(\left|S_{1}\right|^{2}\right) E\left(\left|S_{2}\right|^{2}\right)} \rho \mathrm{e}^{j \theta} \\
\sqrt{E\left(\left|S_{1}\right|^{2}\right) E\left(\left|S_{2}\right|^{2}\right)} \rho \mathrm{e}^{-j \theta} & E\left(\left|S_{2}\right|^{2}\right)
\end{array}\right],
\end{aligned}
$$

where $\rho \mathrm{e}^{j \theta}$ is the complex correlation coefficient of two components in (3).

2.2. The Joint Distribution of Two Multilook Intensities from Different Polarimetric Channels. When only incomplete polarimetric data are available, for instance, the amplitude or intensity of copolarized components (i.e., $\mathrm{HH}$ and VV), the joint distribution of intensity, or amplitude from two correlated polarimetric channels is of importance for constructing signal processing algorithms such as detection and classification in this case. Lee et al. [8] have derived the PDF of joint normalized multilook intensities by integrating (5) with respect to the two off-diagonal elements, which is modeled as

$$
\begin{aligned}
& p_{R_{1}, R_{2}}\left(R_{1}, R_{2}\right) \\
& =\frac{n^{n+1}\left(R_{1} R_{2}\right)^{(n-1) / 2} \exp \left(-n\left(R_{1}+R_{2}\right) /\left(1-\rho^{2}\right)\right)}{\Gamma(n)\left(1-\rho^{2}\right) \rho^{n-1}} \\
& \times I_{n-1}\left(2 n \sqrt{R_{1} R_{2}} \frac{\rho}{1-\rho^{2}}\right), \quad R_{1}, R_{2}, n>0,0<\rho<1,
\end{aligned}
$$

where $I_{n-1}(\cdot)$ is the first type modified Bessel function of order $n-1, R_{i}, i=1,2$, represents normalized multi-look intensity of $i$ th polarimetric channel with the expression $R_{i}=$ $(1 / n) \sum_{k=1}^{n}\left(\left|S_{i}(k)\right|^{2} / E\left(\left|S_{i}\right|^{2}\right)\right)$.

Furthermore, to facilitate the posterior derivation, by the transforms with the following forms:

$$
\begin{aligned}
& B_{1}=n R_{1}, \\
& B_{2}=n R_{2},
\end{aligned}
$$

one can obtain the joint density of $B_{1}$ and $B_{2}$ as (see [8] for details)

$$
\begin{aligned}
& p_{B_{1}, B_{2}}\left(B_{1}, B_{2}\right) \\
& =\frac{\left(B_{1} B_{2}\right)^{(n-1) / 2} \exp \left(-\left(B_{1}+B_{2}\right) /\left(1-\rho^{2}\right)\right)}{\Gamma(n)\left(1-\rho^{2}\right) \rho^{n-1}} \\
& \times I_{n-1}\left(2 \sqrt{B_{1} B_{2}} \frac{\rho}{1-\rho^{2}}\right), \quad B_{1}, B_{2}, n>0,0<\rho<1 .
\end{aligned}
$$




\section{The PMA Detector}

3.1. PMA Detector. In a single-channel SAR image, it is usually assumed that strong backscattering comes from targets. A target point will be lost when the backscattering amplitude or intensity from the target is not large enough compared with the clutter background, generally, due to low signal-toclutter ratio (SCR). In other words, SCR is an essential factor influencing the detection performance when only amplitude or intensity data are available. Thus, the principle designing a good detector should naturally enable SCR to be improved, that is, enhancing target and restraining clutter.

As we know, the span detector is a widely used processor which is a noncoherent sum of all polarimetric channels and only makes use of image intensities. For dual-polarization multilook case, the span is given by [7]

$$
\operatorname{span}=\frac{1}{n} \sum_{k=1}^{n}\left|S_{1}(k)\right|^{2}+\frac{1}{n} \sum_{k=1}^{n}\left|S_{2}(k)\right|^{2} .
$$

This detector can be regarded as the synthetic power of all channels. Consequently, some investigations have shown that a lower noise level and a higher SCR can be obtained by this detector than $\mathrm{HH}, \mathrm{HV}$, or VV individually. This conclusion implies that the way of synthetic power can arrive at the purpose of improving SCR, so that the targets can be more easily discriminated from the clutter, compared with that only arbitrary single-channel information is used. Meanwhile, it is also very hard to adaptively give a proper detection threshold by the span detector due to the unknown knowledge of the corresponding statistics. Motivated by these considerations, we construct a detector in this paper by means of another synthetic power, that is, the product of multilook amplitudes from two polarimetric channels, for convenience, called PMA detector which can be defined as

$$
\xi=\sigma_{1} \sigma_{2} \sqrt{R_{1} R_{2}}=\sigma_{1} \sigma_{2} \frac{\sqrt{B_{1} B_{2}}}{n},
$$

where $\sigma_{i}=E\left(\left|S_{i}\right|^{2}\right)$. From an intuitive understanding, in the variable $\xi$ domain, for the targets, their amplitudes of two polarimetric channels are both larger than the surrounding clutter, which results in a much faster cumulative speed of power for targets verse clutter by multiplying the first image amplitude by the second image one, and, hence, targets' values of $\xi$ are much more prominent and target signal can be enhanced.

3.2. The CFAR Algorithm of PMA Detector. For dual-pol SAR amplitude data, based on the multiplicative model and the assumption that the energy of two channels is balanced, the product of multilook amplitudes from two polarimetric channels can be denoted as

$$
\begin{aligned}
\zeta & =\sqrt{\frac{1}{n} \sum_{k=1}^{n}\left|A_{1} X_{1}(k)\right|^{2} \frac{1}{n} \sum_{k=1}^{n}\left|A_{2} X_{2}(k)\right|^{2}} \\
& =\sqrt{A_{1}^{2} A_{2}^{2} \frac{1}{n} \sum_{k=1}^{n}\left|X_{1}(k)\right|^{2} \frac{1}{n} \sum_{k=1}^{n}\left|X_{2}(k)\right|^{2}}=A_{1} A_{2} \xi,
\end{aligned}
$$

where $A_{i}$ represents the backscattering RCS amplitude component of $i$ th receiving polarimetric channel, and $X_{i}(k)=\left|S_{i}(k)\right|^{2} / E\left(\left|S_{i}\right|^{2}\right)$. Recently, Frery et al. [11] have proposed a well-known $\mathscr{G}^{0}$ distribution to model the clutter regions in SAR images, and the product of multilook amplitudes from two polarimetric channels, that is, the proposed detector in this paper, employs an intensity expression of this distribution with three distinct parameters $\sigma, n$, and $\alpha$, when applying to the modeling of sea background. That is,

$$
p_{\zeta}(\zeta)=\frac{\sigma^{n}}{B(n,-\alpha)} \frac{\zeta^{n-1}}{(1+\sigma \zeta)^{n-\alpha}}, \quad \sigma, n,-\alpha, \zeta>0,
$$

where $B(\cdot, \cdot)$ is the beta function. The estimates $\widehat{\alpha}, \widehat{\sigma}$, and $\widehat{n}$ corresponded, respectively, to the parameters $\alpha, \sigma$, and $n$ can be easily obtained with the help of numerical calculation based on the method of log-cumulants (MoLC) [12] as

$$
\begin{gathered}
\Psi(\widehat{n})-\Psi(-\widehat{\alpha})-\ln (\widehat{\sigma})=\frac{1}{N} \sum_{i=1}^{N}\left[\ln \left(x_{i}\right)\right]=\widehat{\widetilde{c}}_{1}, \\
\Psi(1, \widehat{n})+\Psi(1,-\widehat{\alpha})=\frac{1}{N} \sum_{i=1}^{N}\left[\left(\ln \left(x_{i}\right)-\widehat{\widetilde{c}}_{1}\right)^{2}\right], \\
\Psi(2, \widehat{n})-\Psi(2,-\widehat{\alpha})=\frac{1}{N} \sum_{i=1}^{N}\left[\left(\ln \left(x_{i}\right)-\right)^{3}\right],
\end{gathered}
$$

where $\Psi(\cdot)$ represents the digamma function (i.e., the logarithmic derivative of the gamma function), $\Psi(r, \cdot)$ is the $r$ th order polygamma function (i.e., the $k$ th order derivative of the digamma function), and $\left\{x_{i}\right\}, i \in[1, N]$, is a given sample set.

Given the density shown in (13), its cumulative distribution function (CDF) is written as [11]

$$
F_{\zeta}(x)=\frac{\sigma^{n} x^{n}}{n B(n,-\alpha)}{ }_{2} F_{1}(n-\alpha, n ; n+1 ;-\sigma x),
$$

where ${ }_{2} F_{1}(\cdot, \cdot ; \cdot ; \cdot)$ is the Gauss hypergeometric function. For a given value of the false alarm probability, denoted by $P_{\mathrm{fa}}$, the corresponding CFAR threshold $T$ for the distribution shown in (13) can be obtained from

$$
1-P_{\mathrm{fa}}=F_{\zeta}(T)=\frac{\sigma^{n} T^{n}}{n B(n,-\alpha)}{ }_{2} F_{1}(n-\alpha, n ; n+1 ;-\sigma T) .
$$

Considering that $F_{\zeta}(T)$ is strictly monotonously increasing, the threshold $T$ can be accurately calculated via the numerical solution or a simple bisection method.

\section{Experimental Results and Analysis}

The test dual-pol SAR amplitude data used in this study are a large TerraSAR-X StripMap mode geocoded scene over Nanjing, China, acquired with high-resolution $6 \mathrm{~m} \times 6 \mathrm{~m}$ (azimuth $\times$ range) and $\mathrm{HH}$-polarization and VV-polarization. Figure 1(a) provides a fake-color image of 


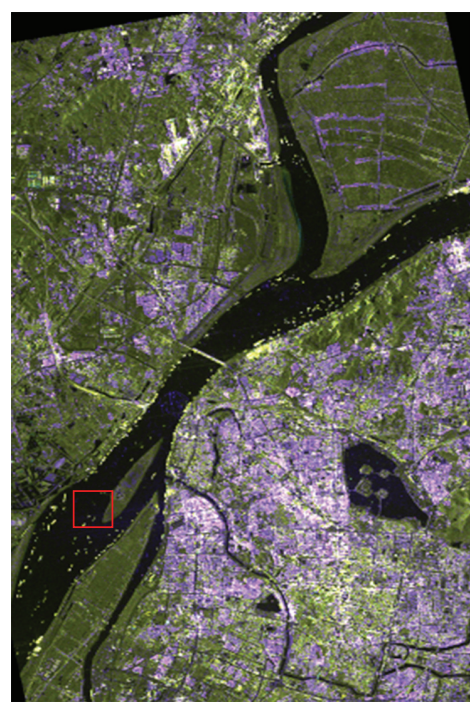

(a)

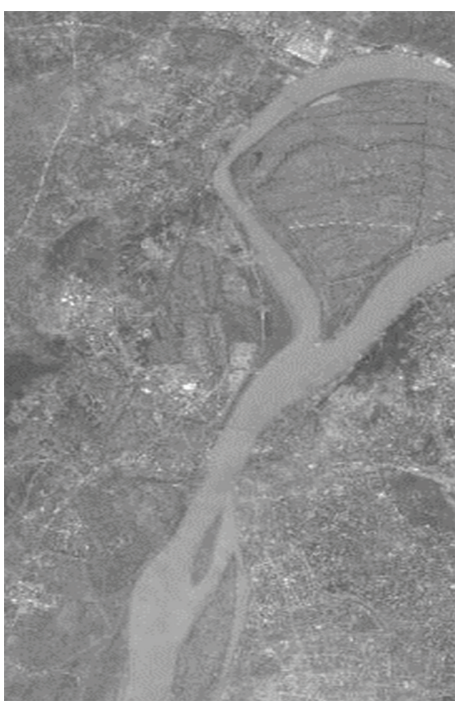

(b)

FIgure 1: The image of Nanjing: (a) TerraSAR-X image; (b) SPOT5 optical image.

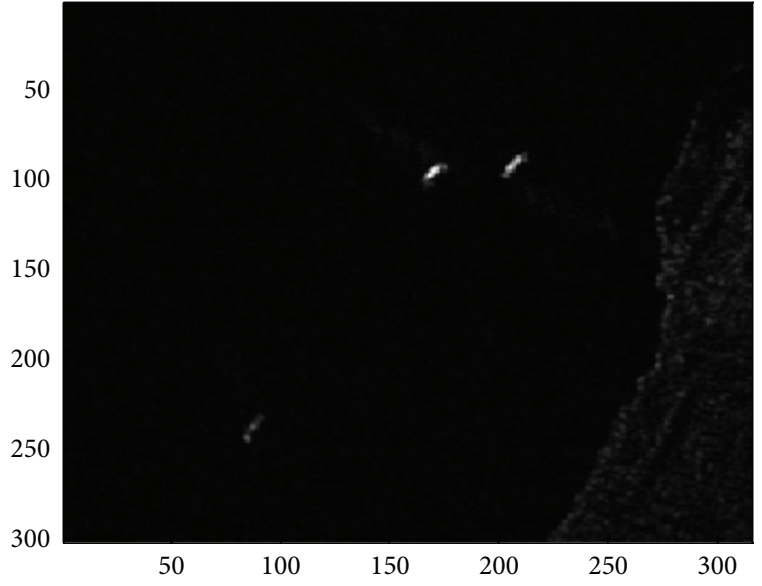

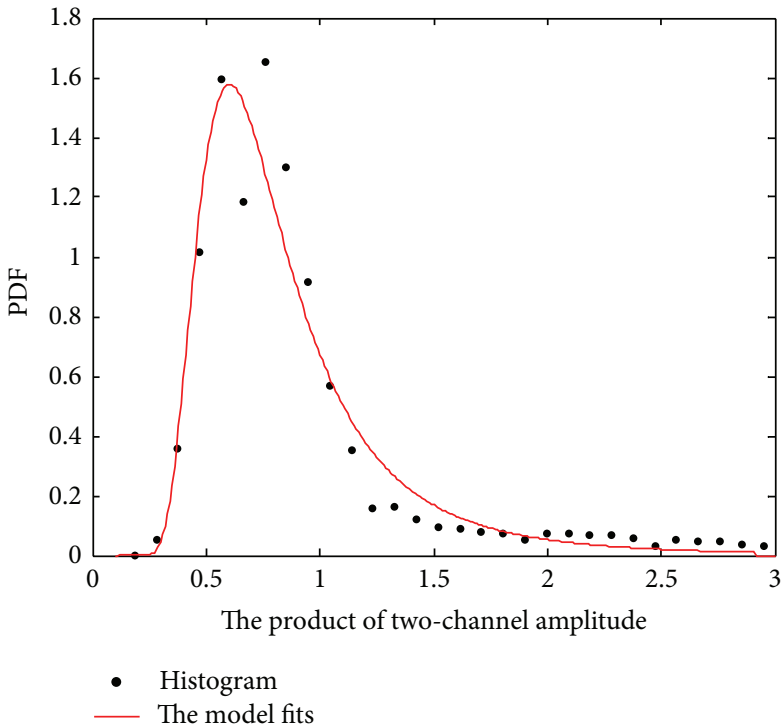

(b)

FIGURE 2: The ship chip: (a) the product image; (b) the fitting result.

this scene. The horizontal and vertical axes are the directions of azimuth and range, respectively. Meanwhile, in order to make a visible comparison, Figure 1(b) gives an optical (ground truth) remote sensing photograph of the test site from SPOT5 satellite.

The red rectangle box region shown in Figure 1(a) consisting of several ships and sea clutter is our investigating area. The product image of multilook amplitudes from two polarimetric channels is shown in Figure 2(a). Figure 2(b) shows the fitting results of the distribution in (13) for the product image of the area indicated in the rectangle box in Figure 2(a). The parameters $n, \alpha$, and $\sigma$ are estimated to $\hat{n}=$ $100.5744, \widehat{\alpha}=-1.7851$, and $\widehat{\sigma}=120.7748$, respectively. As shown in Figure 2(b), it is clear that the fitting result agrees well with the theoretical distribution.

Furthermore, giving the theoretical false alarm probability $P_{\mathrm{fa}}=10^{-8}$, the detection results are shown in Figure 3 . It is easy to observe that all ships are detected, whilst a false alarm occurs, which proves the effectiveness of CFAR detection method based on PMA detector for ships.

\section{Conclusion}

Aiming at the adaptive detection of a ship when only high-resolution dual-polarization SAR amplitude data are 


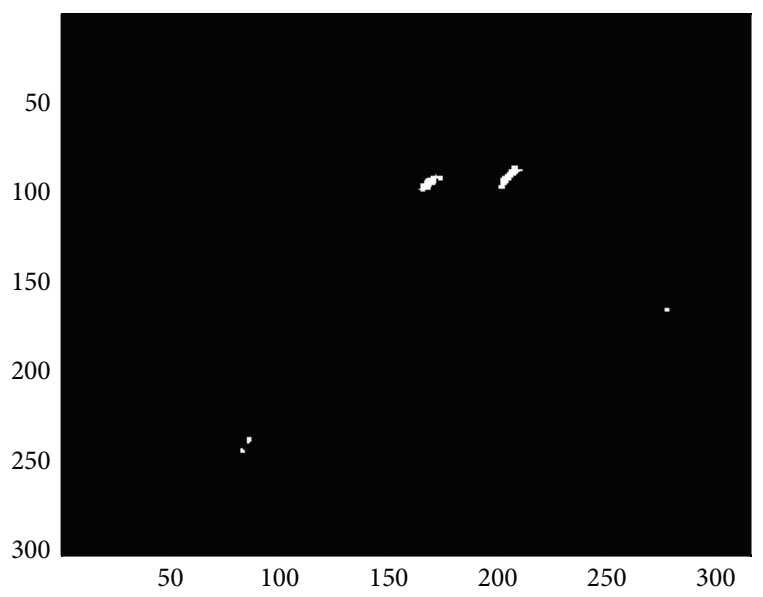

FIgURE 3: The ship detecting result.

available, a CFAR detecting method has been proposed in this paper. We first design a novel PMA detector, which can improve the signal-to-clutter ratio (SCR) and make the discrimination of a ship from clutter more easily. Meanwhile, the PMA detector's statistical model has been described by the well-known $\mathscr{G}^{0}$ distribution when facing complex sea background. The experiments performed on measured dual-polarization TerraSAR-X images demonstrate the good performance of the proposed CFAR detecting method.

\section{Acknowledgment}

The author would like to appreciate the National Natural Science Foundation of China for the support under Grant no. 41171316.

\section{References}

[1] D. J. Crisp, The State-of-the-Art in Ship Detection in Synthetic Aperture Radar Imagery, DSTO, Departement Defence, Australian Government, Canberra, Australia, Public Release Document DSTO-RR-0272, 2004.

[2] C. J. Oliver and S. Quegan, Understanding Synthetic Aperture Radar Images, Artech House, Norwood, Mass, USA, 1998.

[3] I. C. Sikaneta, Detection of ground moving objects with synthetic aperture radar [Ph.D. thesis], University of Ottawa, 2002.

[4] F. T. Ulaby and C. Elachi, Radar Polarimetric for Geoscience Application, Artech House, Boston, Mass, USA, 1990.

[5] C. E. Livingstone and A. A. Thompson, "The moving object detection experiment on RADARSAT-2," Canadian Journal of Remote Sensing, vol. 30, no. 3, pp. 355-368, 2004.

[6] V. A. Krylov, G. Moser, S. B. Serpico, and J. Zerubia, "Supervised high-resolution dual-polarization SAR image classification by finite mixtures and copulas," IEEE Journal on Selected Topics in Signal Processing, vol. 5, no. 3, pp. 554-566, 2011.

[7] R. D. Chaney, M. C. Bud, and L. M. Novak, "On the performance of polarimetric target detection algorithms," IEEE Aerospace and Electronic Systems Magazine, vol. 5, no. 11, pp. 10-15, 1990.

[8] J. S. Lee, K. W. Hoppel, S. A. Mango, and A. R. Miller, "Intensity and phase statistics of multilook polarimetric and interferometric SAR imagery," IEEE Transactions on Geoscience and Remote Sensing, vol. 32, no. 5, pp. 1017-1028, 1994.

[9] C. H. Gierull, "Statistics of SAR interferograms with application to moving target detection," Tech. Rep. 2001-045, Defense Research Establishment Ottawa, Department of National Defense, Ottawa, Canada, 2001.

[10] N. R. Goodman, "Statistical analysis based on a certain multivariate complex gaussian distribution (an introduction)," Annals of Mathematical Statistics, vol. 34, no. 152, pp. 152-180, 1963.

[11] A. C. Frery, J. Jacobo-Berlles, J. Gambini, and M. E. Mejail, "Polarimetric SAR image segmentation with B-splines and a new statistical model," Multidimensional Systems and Signal Processing, vol. 21, no. 4, pp. 319-342, 2010.

[12] J. M. Nicolas, "Introduction to second kind statistic: application of log-moments and log-cumulants to SAR image law analysis," Traitement du Signal, vol. 19, no. 3, pp. 139-167, 2002. 

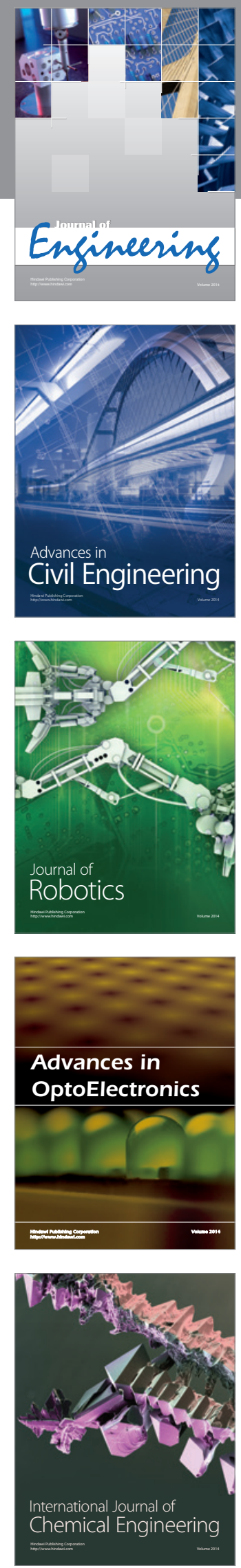

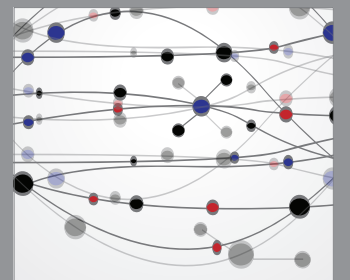

The Scientific World Journal
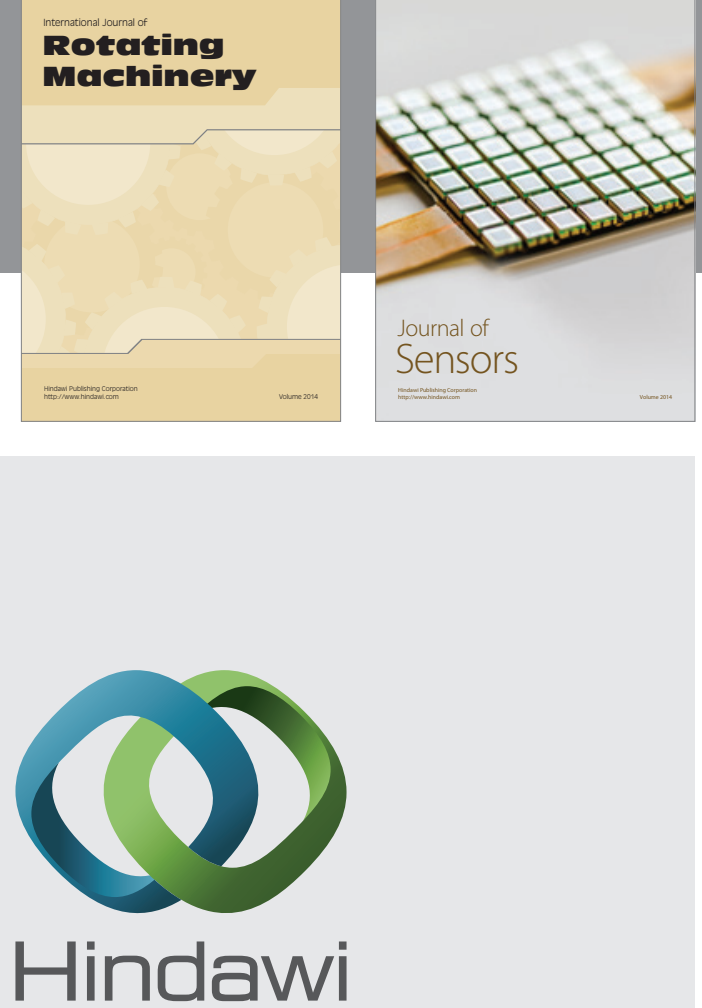

Submit your manuscripts at http://www.hindawi.com
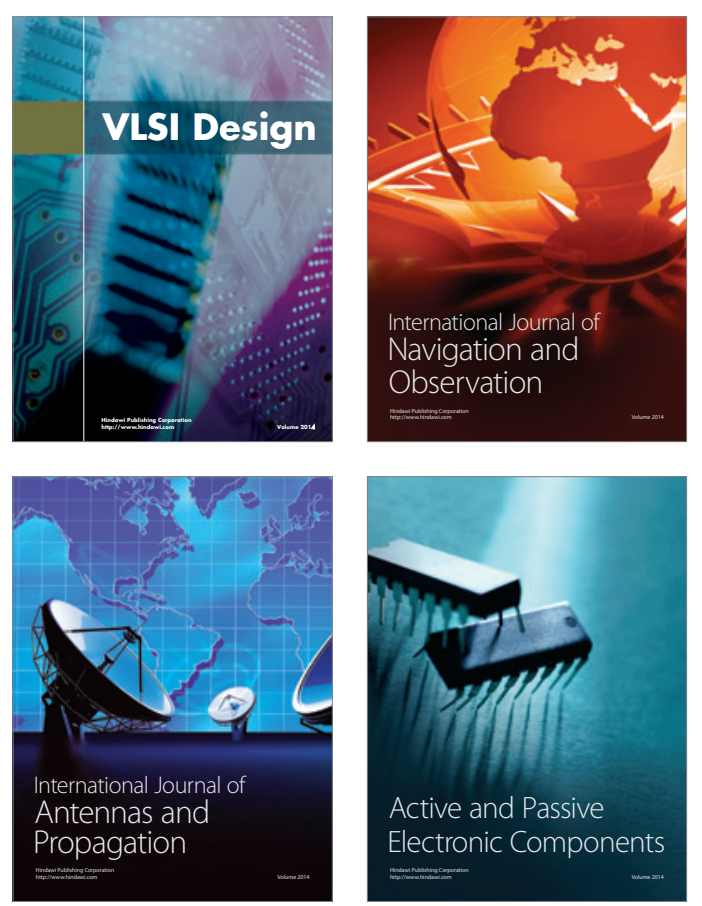
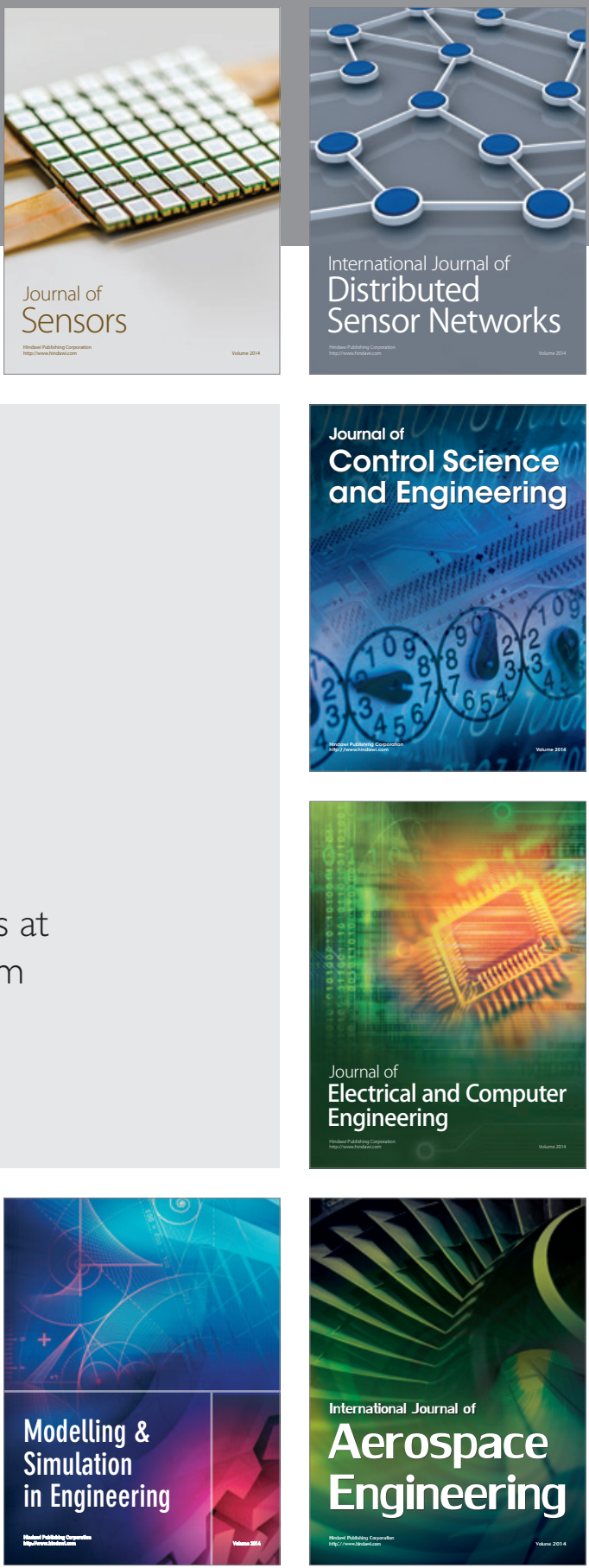

Journal of

Control Science

and Engineering
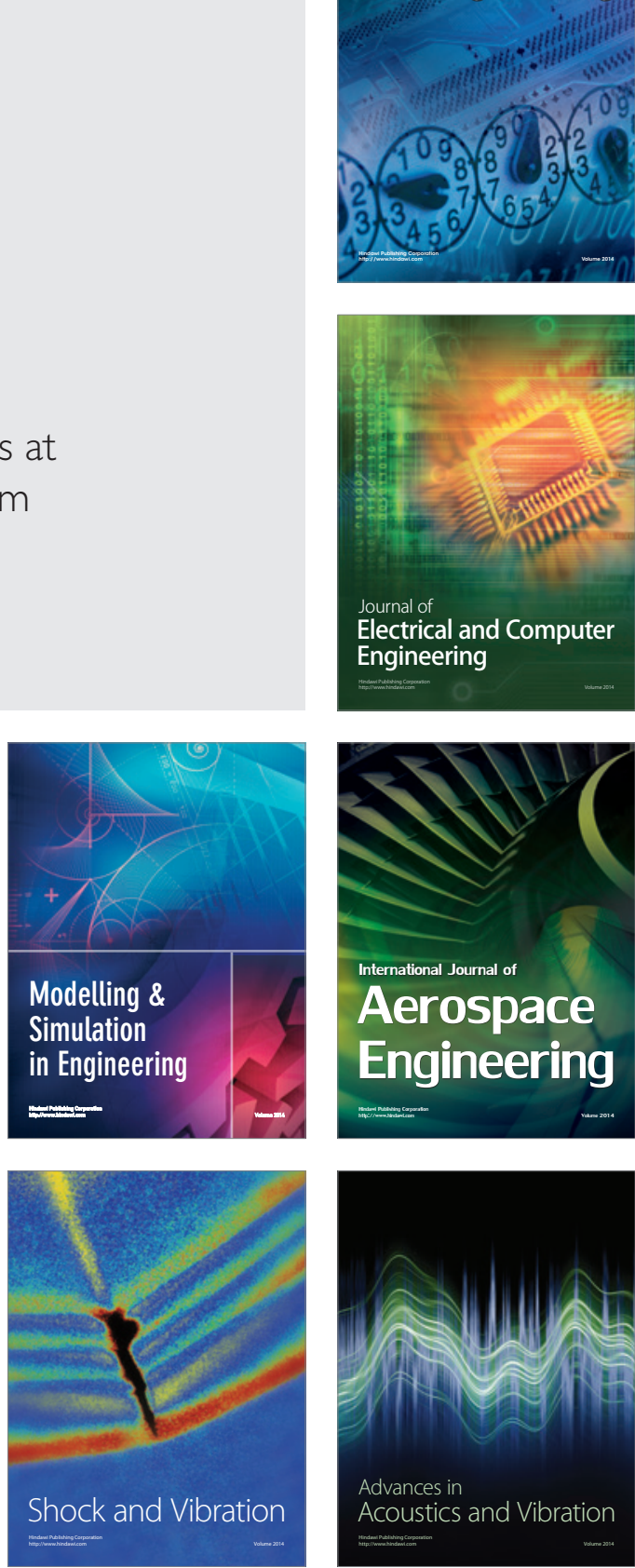